Mode of Death in Octogenarians Treated With Cardiac Resynchronization Therapy

Peer-reviewed author version

MARTENS, Pieter; VERBRUGGE, Frederik; NIJST, Petra; DUPONT, Matthias \& MULLENS, Wilfried (2016) Mode of Death in Octogenarians Treated With Cardiac

Resynchronization Therapy. In: JOURNAL OF CARDIAC FAILURE, 22(12), p. 970-977.

DOI: 10.1016/j.cardfail.2016.09.023

Handle: http://hdl.handle.net/1942/23236 


\section{Mode of Death in Octogenarians Treated with Cardiac Resynchronization Therapy}

Original Article

Pieter Martens M.D. ${ }^{1,2}$, Frederik H. Verbrugge M.D. Ph.D. ${ }^{1}$, Petra Nijst M.D. ${ }^{1,2}$

Matthias

\section{Dupont M.D. ${ }^{1}$, Wilfried Mullens M.D. Ph.D. ${ }^{1,3}$}

1. Department of Cardiology, Ziekenhuis Oost-Limburg, Genk, Belgium

2. Doctoral School for Medicine and Life Sciences, Hasselt University, Diepenbeek, Belgium.

3. Biomedical Research Institute, Faculty of Medicine and Life Sciences, Hasselt University, Diepenbeek, Belgium.

Grant Support: Pieter Martens, Petra Nijst, and Wilfried Mullens are researchers for the Limburg Clinical Research Program (LCRP) UHasselt-ZOL-Jessa, supported by the foundation Limburg Sterk Merk (LSM), Hasselt University, Ziekenhuis Oost-Limburg and Jessa Hospital.

\section{Corresponding author:}

Wilfried Mullens, MD, Ph.D.

Department of Cardiology, Ziekenhuis Oost-Limburg

Schiepse Bos 6, 3600 Genk, BELGIUM

Tel: +32 89327160 | Fax: +32 89327918 | E-mail: Wilfried.Mullens@zol.be 


\section{Abbreviations}

CRT: Cardiac resynchronization therapy

HFrEF: Heart failure with reduced ejection fraction

CRT-D: Cardiac resynchronization therapy - Defibrillator

CRT-P: Cardiac resynchronization therapy - Pacemaker

LVEF: left ventricular ejection fraction

LVEDD: left ventricular end-diastolic diameter

SCD: Sudden cardiac death 


\section{Abstract \\ BACKGROUND}

Cardiac resynchronization therapy (CRT) improves morbidity and mortality in heart failure with reduced ejection fraction (HFrEF) and electrical dyssynchrony. CRT patients in clinical practice are older compared to clinical trials.

\section{OBJECTIVE}

To investigate clinical response, reverse remodeling, outcome and mode of death in octogenarians receiving $\mathrm{CRT}$.

\section{METHODS}

Baseline characteristics, change in New York Heart Association (NYHA) functional class, reverse ventricular remodeling, heart failure readmissions, all-cause mortality and mode of death were evaluated in CRT patients with comparison between octogenarians and non-octogenarians. In addition, annual mortality rates of octogenarians undergoing CRT were compared with age-matched controls from the general population using national actuarial tables.

\section{RESULTS}

A total of 686 patients, including 178 octogenarians (26\%), were followed for $38 \pm 22$ months. Octogenarians exhibited a similar change in NYHA functional class $(P=0.640)$, left ventricular ejection fraction increase $(P=0.796)$ and decrease in end-diastolic $(P=0.441)$ and end-systolic $(P=0.312)$ diameter compared to their younger counterparts undergoing CRT. Octogenarians had a higher allcause mortality risk $(\mathrm{P}<0.001)$, but heart failure readmission risk did not differ $(\mathrm{HR}=0.916 ; \mathrm{Cl}=0.638$ 1.313; $\mathrm{P}=0.632$ ). A higher proportion of non-cardiac deaths was observed in octogenarians (74\%) versus younger patients $(50 \% ; \mathrm{P}=0.022)$, with worsening heart failure rather than malignant tachyarrhythmia being the main cardiac cause of death. Compared to an age-matched sample from the general population, octogenarians receiving CRT had an equivalent annual mortality rate (Log-

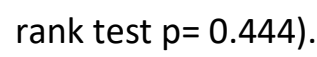

\section{CONCLUSION}

Octogenarians retain the ability to mount a significant symptomatic and ventricular remodeling response after CRT, resulting in survival similar to the general age matched population. 


\section{Introduction}

Cardiac resynchronization therapy (CRT) improves symptoms and survival in patients with electrical dyssynchrony and persistent heart failure with reduced ejection fraction (HFrEF) despite optimal medical therapy ${ }^{1 ; 2}$. Notably, the average age of patients included in pivotal CRT trials (62-67 years) differs markedly from the real-world situation ${ }^{3-7}$. In the European CRT Survey, mean age at implantation was 71 years, with $31 \%$ of patients being older than $75^{8}$. With the prevalence of heart failure in octogenarians ranging high at $12-20 \%$, there is a pressing need for more outcome data with CRT in this age group ${ }^{9}$. It is increasingly being recognized that CRT elicits similar symptomatic relief and reverse ventricular remodeling in octogenarians compared to their younger counterparts ${ }^{10 ; 11}$. However, a survival benefit with CRT has not been demonstrated convincingly due to the low number of octogenarians included in randomized controlled trials. Subgroup analyses of randomized CRT trials lack power to detect meaningful differences and may represent highly selected groups of octogenarians that are probably not reflective of the real-world ${ }^{12}$. A recent analysis from the National Cardiovascular Disease Registry - Implantable cardio defibrillator (ICD) registry did demonstrate a survival benefit in octogenarians receiving a CRT-defibrillator (CRT-D) versus ICD ${ }^{13}$. This observation of mortality benefit of CRT-D over ICD has been confirmed in the Israeli ICD-registry

14. However, no patients receiving a CRT-pacemaker (CRT-P) were included in these analyses and the mode of death was not presented. Perhaps the benefit for octogenarians in these registries is more driven by resynchronization and improved heart failure status than protection against malignant ventricular arrhythmias. Attaining clarity about the mode of death in elderly patients is crucial, as it may guide appropriate device selection (i.e., CRT-P versus CRT-D). This study aims to confirm previous findings of improvement in functional and echocardiographic parameters. Furthermore, we aim to determine the mode of death in octogenarians undergoing CRT versus non-octogenarians, and to determine differences in patterns of mode of death that could help steer decision making on device types. 


\section{Methods}

\section{Study design}

Consecutive HFrEF patients undergoing CRT implantation at a single tertiary care center (Ziekenhuis Oost-Limburg, Genk, Belgium) between October, 2008, and August, 2015, were evaluated. CRT indication was in compliance with the European Society of Cardiology guidelines ${ }^{1,15}$. In brief, patients exhibited symptoms of heart failure and a persistently reduced left ventricular reduced ejection fraction (LVEF) below 30-35\% despite optimal medical therapy. As indications for CRT changed over time, initially the focus was more on QRS $>120$ msec (2008 guidelines) but later during the course of the study patients received CRT if QRS $>120 \mathrm{msec}$ with coinciding left bundle branch block morphology or $>150 \mathrm{msec}$ in case of non-left bundle branch block morphology (2012 guidelines) 1;15. Furthermore European guidelines in comparison to American guidelines emphasize the use of CRT-P over CRT-D in patients with very advanced heart failure, chronic kidney disease, cachexia, frailty and multiple or severe co-morbidities ${ }^{16 ; 17}$. The patient's age at the time of implantation was systematically registered and used as an entry criterion for the current analysis. Following implantation, all patients underwent a standardized follow-up schedule and dedicated CRT optimization protocol, as previously published by our group ${ }^{18 ; 19}$. Briefly, all patients received individualized optimization of heart failure care including up-titration of neurohormonal blockers as well as echocardiography-guided optimization of their device settings one day after implantation. Patients in sinus-rhythm received AV-delay optimization using the pulse wave mitral valve inflow pattern ${ }^{20}$. If atrial fibrillation was present only VV-delay optimization was performed. This was repeated as indicated at each visit to a dedicated multidisciplinary CRT clinic. Patients received a first follow-up appointment 6 weeks after implantation and a second after 6 months. Afterwards followup intensity was diminished to once every nine months if patients were deemed stable. Octogenarians versus younger patients were first compared, with subsequent comparison to an agematched sample from the general population. A random dataset of 1,000 age-matched octogenarians was generated based upon the results of the reported annual mortality rate in the 
Belgian national actuarial life tables (Supplementary Appendices) ${ }^{21}$. This study is in compliance with the Declaration of Helsinki. Given its retrospective nature, the need for written informed consent was waived by the local ethical committee. The manuscript was drafted according to the STROBEstatement for observational studies ${ }^{22}$.

\section{Response to cardiac resynchronization therapy}

Response to CRT was systematically assessed after 6 months. Symptomatic response was measured as the change in New York Heart Association (NYHA) functional class between baseline and six month follow-up. Cardiac reverse remodeling was evaluated by comprehensive 2-dimensional echocardiography exams (Philips Medical Systems, iE33w) performed by experienced cardiac sonographers. Reverse ventricular remodeling was defined by the change in LVEF as well as left ventricular end-diastolic (LVEDD) and end-systolic diameter (LVESD) between baseline and six month follow-up. All reported echocardiography measurements were averaged from 3 consecutive cycles (or 5 if atrial fibrillation was present) and assessed as recommended by the American Society of Echocardiography ${ }^{23}$. Left ventricular end-diastolic and end-systolic diameters were measured from the parasternal long axis view. Left ventricular ejection fraction was obtained using the modified Simpson's biplane method in the apical 2- and 4-chamber views.

\section{Clinical end-points}

The total number of heart failure events was registered as well as the time to a first event. Heart failure events were defined as an episode of clinical congestion lasting more than 24-hours and necessitating hospitalization and intravenous diuretics. Patients' vital status were retrieved from the hospital's electronic medical record. This system is linked to a national death registry therefore also capturing out of hospital death. Both heart failure events and all-cause mortality were reported separately and not as a combined endpoint because all-cause mortality in octogenarians was also compared to an age-matched population. 


\section{Mode of death}

The mode of death was categorized according to a modified Hinkle-Thaler classification ${ }^{24}$. Briefly, mode of death was categorized into two groups: cardiac death or non-cardiac death. The cardiac group was further categorized into sudden cardiac death (SCD), worsening heart failure, tachyarrhythmic death and post-cardiac procedure mortality. SCD was defined as a sudden electromechanical dissociation with absence of a shockable rhythm at time of first medical contact and absence of progressive cardiac deterioration. Worsening heart failure included both demise due to congestion and progressive cardiogenic shock. The non-cardiac group was further categorized as cancer, pulmonary disease, infectious disease, gastrointestinal disease, renal-related and neurologic conditions. Mode of death was retrieved from the hospital electronic medical record. In case of missing data the primary care physician or next of kin was contacted by phone. In cases that this stepwise approach did not retrieve the mode of death, the patient was excluded from the analysis.

\section{Statistics}

Continuous variables were expressed as mean \pm standard deviation if normally distributed or median (interquartile range) if normal distribution was absent. Normality was checked by the Shapiro-Wilk statistic. Categorical data were expressed as percentages and compared with the Pearson $\chi^{2}$-test when a large sample size $(n>100)$ was present, or Fisher's exact test when a small sample size was present. Continuous variables were compared with the Student's $t$-test or Mann-Whitney $U$ test as appropriate. The Kaplan-Meier method was used to construct survival curves, with the log-rank test used for comparison among groups. The hazard for heart failure readmission was calculated using Cox-proportional hazard models with reporting of the hazard ratio and the associated $95 \%$ confidence interval. Statistical significance was set at a 2 -tailed probability level of $<0.05$. All statistics were performed using SPSS version 22 (IBM, Chicago, IL). 


\section{Results}

\section{Baseline characteristics of the study population}

A total of 706 patients underwent CRT implantation between October 2008 and August 2015. Twenty patients were excluded because 6 month follow-up appointment had not yet passed, generating a final study population of 686 patients compromising 178 octogenarians (26\%, mean age 83 years) and 508 non-octogenarians (74\%, mean age 69 years) with complete data available for analysis. The baseline characteristics are summarized in Table 1. Octogenarians were more likely to have ischemic heart disease and more often received a CRT-P device. Octogenarians had less dilated left ventricles before implantation, despite similar LVEF compared to non-octogenarians at baseline. The distribution of comorbid conditions is depicted in Table 2. Octogenarians more frequently suffered from atrial fibrillation, a history of hypertension, chronic kidney disease, anemia and cachexia. On average, octogenarians had 6 (interquartile range 3-6) comorbidities in comparison to 4 (interquartile range $3-6)$ in younger patients undergoing CRT $(p<0.001)$. Octogenarians were also more often upgraded from a pacemaker to CRT. The median time between pacemaker implantation and upgrade to CRT was 60.5 months (Inter-quartile range $42-76$ months). Octogenarians received less angiotensin-converting enzyme inhibitors, angiotensin receptor blockers and mineralocorticoid receptor antagonists before implantation, perhaps reflecting the higher burden of chronic kidney disease in this population.

\section{Symptomatic response and reverse ventricular remodeling}

Improvement of NYHA functional class after 6 months could be assessed in 172 octogenarians (97\%) and 481 non-octogenarians (95\%) with similar improvement in both groups $(P=0.640)$, see figure 1 . Seventy-three percent of octogenarians improved at least one NYHA functional class versus $72 \%$ of the non-octogenarians. Twenty-two percent of octogenarians versus $25 \%$ of non-octogenarians improved two or more NYHA functional classes. Full echocardiographic data were available in 169 octogenarians (96\%) and 473 non-octogenarians (93\%). Both octogenarians and non-octogenarians 
demonstrated a significant increase in LVEF $(+12.9 \pm 11.5 \%$ vs. $12.6 \pm 13.2 \%$, respectively; $P=0.796)$ and decrease in LVEDD $(-0.4 \pm 1.5 \mathrm{~cm}$ vs. $-0.5 \pm 1.3 \mathrm{~cm} ; \mathrm{P}=0.441)$ and LVESD $(-0.6 \pm 0.8 \mathrm{~cm}$ vs. $-0.4 \pm 1.1 \mathrm{~cm}$; $p=0.312)$, which was comparable in both groups. Octogenarians with $(n=55)$ versus without $(n=123)$ atrial fibrillation had similar percentage of biventricular pacing (91\% respectively $93 \%$ of patients had $>99 \%$ biventricular pacing; $p=0.685$ ). Patients with atrial fibrillation had similar positive reverse remodeling whether octogenarians or non-octogenarians.

\section{Heart failure readmissions}

Complete data on heart failure readmissions were available in 174 octogenarians (98\%) and 502 nonoctogenarians (99\%). Mean follow-up was $38 \pm 22$ months. Forty-seven heart failure readmissions occurred in the octogenarians group compared to 92 events in non-octogenarians ( $P=0.077)$. The median time (interquartile range) to a first heart failure readmission was 266 days ( $120-649$ days) in octogenarians versus 243 days (106 - 478 days) in younger patients ( $P=0.212)$. Octogenarians showed no difference in risk for heart failure readmissions in comparison to non-octogenarians (Hazard ratio 0.916, confidence interval $=0.638-1.313, \mathrm{P}=0.632$ ).

\section{Survival and mode of death}

During a mean follow-up period of 38 months, a total of 113 (16.5\%) patients died. Full outcome data were available in 175 octogenarians (98\%) and 503 non-octogenarians (99\%). Forty-one octogenarians died (23\%) versus 72 patients in the younger group (14\%; $\mathrm{P}<0.001$ for the difference between groups). Figure 2 illustrates the survival of octogenarians compared to younger patients and versus aged-matched octogenarians from the general population. Compared to this latter group, octogenarians undergoing CRT had a similar annual mortality rate $(P=0.444)$. Figures 3 presents the mode of death in the octogenarians and non-octogenarians undergoing CRT. In total 100 patients (88.5\%) had a mode of death available for analysis. The mode of death was retrieved from the electronic hospital record with the international classification of disease (ICD-10) code used to 
categorize mode of death in 90 patients. In the other 10 patients mode of death was retrieved from the primary care physician records $(n=9)$ and in 1 case by direct contact with the next of kin. In the remaining thirteen patients (11.5\%) the mode of death could not be retrieved. Compared to their younger counterparts, octogenarians were more likely to die from a non-cardiac cause of death ( $74 \%$ versus $50 \%, \mathrm{P}=0.022$ ). In both groups heart failure was the most common cardiac cause of death ( $n=15$ in non-octogenarians and $n=9$ in octogenarians). Cancer was the most frequent non cardiac cause of death in non-octogenarians ( $n=13)$ versus infectious diseases in octogenarians ( $n=14)$.

\section{Discussion}

This retrospective analysis of a large contemporary CRT population adds important knowledge to the increasing body of evidence regarding biventricular pacing in elderly patients. The current results confirm previous reports that octogenarians exhibit similar symptomatic and ventricular remodeling responses to $\mathrm{CRT}{ }^{10 ; 11}$. In addition, this analysis provides an in-depth analysis of the mode of death in octogenarians receiving CRT. Importantly, octogenarians were most likely to die from a non-cardiac condition. Additionally, worsening heart failure rather than malignant tachyarrhythmia was the main cardiac cause of death. This observation strengthens the notion that implanting CRT-P devices in elderly patients is an appropriate practice ${ }^{16}$. Furthermore, this analysis suggests that elderly HFrEF patients receiving CRT for an appropriate guideline-recommended indication may demonstrate a life expectancy similar to the general age-matched population.

An aging population and rising prevalence of chronic HFrEF are vastly shaping the medical landscape, placing financial burdens on society ${ }^{25}$. Adoption of cost-efficient class I guideline recommended live-saving therapies is essential from a therapeutic and preventive perspective to lessen the burden of heart failure. Despite the well-established benefits of CRT in randomized controlled trials, only limited data is available pertaining to the benefit of CRT in octogenarians due to an age threshold in these trials ${ }^{3-7 ; 26}$. In the current analysis, octogenarians had similar symptomatic 
improvement, reverse ventricular remodeling as well as a similar readmission rate compared to their younger counterparts. As a result, octogenarians and non-octogenarians experienced a similar median time spent out of hospital after CRT implantation to first heart failure admission. This observation carries a strong clinical implication because time spent out of hospital directly correlates with quality of life from a patient perspective and economic gain from a legislative perspective.

The choice of CRT device with or without defibrillator has been a contentious topic due to the lack of direct comparison ${ }^{27}$. The only large randomized controlled trial directly comparing CRT-D, CRT-P and medical therapy (COMPANION) was not powered to examine differences in mortality between CRT-P and CRT-D ${ }^{4}$. Indeed, the absence of consensus regarding the choice of CRT-P versus CRT-D has resulted in considerable differences in geographical implantation practices, with CRT-D dominating in the United States of America while CRT-P devices are more commonly implanted in Europe. This lack of consensus is reflected in current guidelines, with absence of specific recommendations but merely guidance for selection of CRT-P versus CRT-D ${ }^{16}$. Indeed, CRT-P is suggested to be preferred over CRT-D in patients with very advanced heart failure, chronic kidney disease, cachexia, frailty and multiple or severe co-morbidities. Understanding the mode of death is perhaps essential for teasing out which patients benefit most from which device-based therapy. Though the benefit of CRT-D in aborting tachyarrhythmic death in comparison to CRT-P is obvious, it comes at an incremental cost, more complex device technology and risk for inappropriate therapy. Also, elderly patients might opt for improvement in quality of life more than expanding life span. In that way, defibrillator therapy might prevent them from dying suddenly, which is often perceived as the preferred mode of death in this population ${ }^{28}$. In the current study population, non-cardiac death was the main driver of mortality in octogenarians, thereby significantly differing from the higher proportion of cardiac death in non-octogenarians. Moreover, even in octogenarians cardiac death was mainly due to worsening heart failure, therefore suggesting limited additional benefit of adding a defibrillator function. Indeed, primary and even secondary prevention ICD-studies have suggested little added value of ICD-therapy in the elderly ${ }^{29 ; 30}$. Furthermore, a high prevalence of comorbidities 
has been linked to a decreased value of ICDs, perhaps reflecting the competing risk between arrhythmic death and non-arrhythmic death in the elderly with heart failure ${ }^{31}$. In our cohort octogenarians indeed had higher burden of comorbidities in comparison to their younger counterparts. Results of the current analysis are in line with the large prospective CERTITUDE cohort from France ${ }^{32}$. In CERTITUDE, CRT-P was more commonly applied in older patients, patients with advanced disease (NYHA IV), atrial fibrillation and chronic kidney disease. Despite the finding that CRT-P was associated with an increased risk of death in comparison to CRT-D, the mode of death analysis revealed deaths for which adding a defibrillator function holds little promise. Although this cohort did not specifically assess octogenarians, the findings are in line with the current analysis. Taken together, the data indicate that CRT is effective in improving symptomatic status and reducing heart failure burden in the elderly. However, the mode of death and comparison with age-matched population suggests that mortality is mainly driven by non-cardiac conditions for which ICD-therapy is of little added value.

\section{Limitations}

Several limitations should be taken into consideration in interpreting this study. First, this was a retrospective study. However, patients were followed in a dedicated CRT clinic with systematic follow-up, making follow-up similar for all patients, and almost complete data were available for the endpoint analysis. Second, although our population is a real world population rather than a randomized trial population, octogenarians included in this study who underwent CRT are probably not truly reflective of the general age-matched population because of clinical selection bias. Third, some of the endpoints such as heart failure readmission might be underpowered, therefore the results are mainly hypothesis generating. Finally, no baseline characteristics of the age-matched controls are available in actuarial tables. Therefore, it is difficult to determine to what extent this population differs from the octogenarians in this study, especially with regards to comorbidity burden. 


\section{Conclusion}

Benefits regarding symptomatic improvement and reverse ventricular remodeling response are well retained in elderly patients undergoing CRT. This translates into similar proportions of octogenarians and younger patients admitted for heart failure and an equal time spent out of the hospital prior to first heart failure admission. The predominance of non-cardiac death in octogenarians suggests that the conventional practice in Europe of implanting predominantly CRT-P in elderly patients with comorbidities is justifiable. Despite a difference in survival between octogenarians and younger patients undergoing CRT, the former had a similar survival compared to age-matched subjects from the general population. 


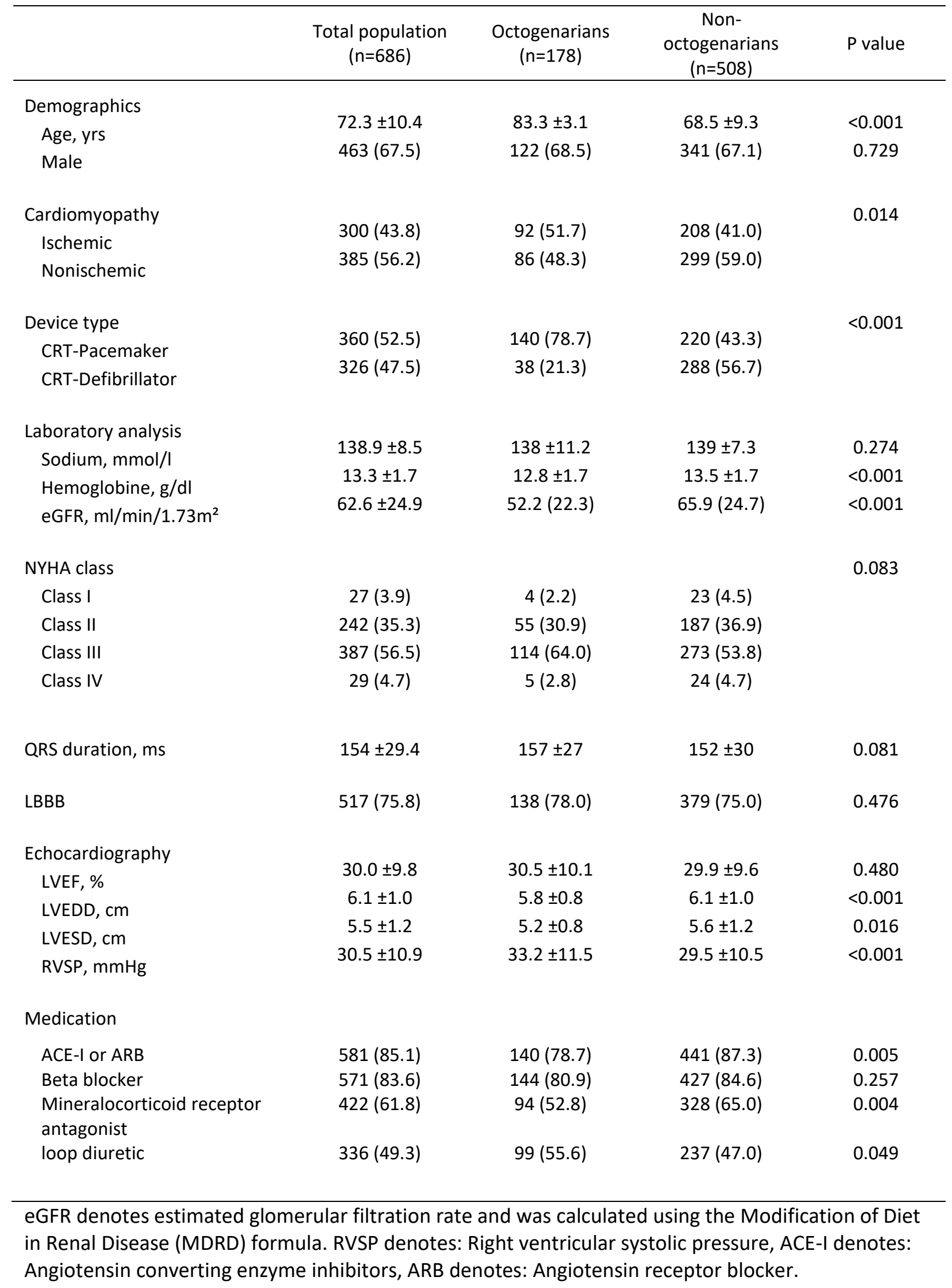


Table 2: Distribution of comorbidities

\begin{tabular}{lcccc}
\hline & $\begin{array}{c}\text { Total population } \\
(\mathrm{n}=686)\end{array}$ & $\begin{array}{c}\text { Octogenarians } \\
(\mathrm{n}=178)\end{array}$ & $\begin{array}{c}\text { Non- } \\
\text { octogenarians } \\
(\mathrm{n}=508)\end{array}$ & P value \\
\hline Atrial fibrillation & $255(37.2)$ & $89(50.0)$ & $166(32.7)$ & $<0.001$ \\
COPD & $116(16.9)$ & $31(17.4)$ & $85(16.8)$ & 0.842 \\
History of hypertension & $547(79.7)$ & $153(86.0)$ & $394(77.6)$ & 0.016 \\
Previous Stroke & $49(7.2)$ & $13(7.3)$ & $36(7.1)$ & 0.933 \\
Dyslipidemia & $480(70.0)$ & $130(73.0)$ & $350(68.9)$ & 0.300 \\
Diabetes & $181(26.4)$ & $54(30.4)$ & $127(25.0)$ & 0.164 \\
CKD & $313(45.6)$ & $116(65.2)$ & $197(38.8)$ & $<0.001$ \\
Anemia* & $207(30.5)$ & $80(45.2)$ & $127(25.3)$ & $<0.001$ \\
Cachexia & $26(3.8)$ & $12(6.7)$ & $14(2.8)$ & 0.017 \\
Pre-implant Pacemaker & $133(19.4)$ & $44(24.7)$ & $89(17.6)$ & 0.038 \\
Pre-implant ICD & $58(8.5)$ & $11(6.2)$ & $47(9.3)$ & 0.203 \\
Previous valvular surgery & $94(13.7)$ & $24(13.5)$ & $70(13.8)$ & 0.914 \\
CABG & $132(19.3)$ & $36(20.2)$ & $96(18.9)$ & 0.707 \\
Previous PCl & $217(31.7)$ & $74(41.6)$ & $143(28.3)$ & 0.001 \\
\hline
\end{tabular}

$¥$ : CKD was defined as a eGFR below $60 \mathrm{ml} / \mathrm{min} / 1.73 \mathrm{~m}^{2}$ calculated using the Modification of Diet in Renal Disease (MDRD) formula, anemia was defined according to WHO-criteria (female Hgb below 12 $\mathrm{g} / \mathrm{dl}$, male Hgb below $13 \mathrm{~g} / \mathrm{dl}$ ), a: cachexia was defined as a BMI below 18 . 
Figure 1: Change in NYHA functional class after 6 months

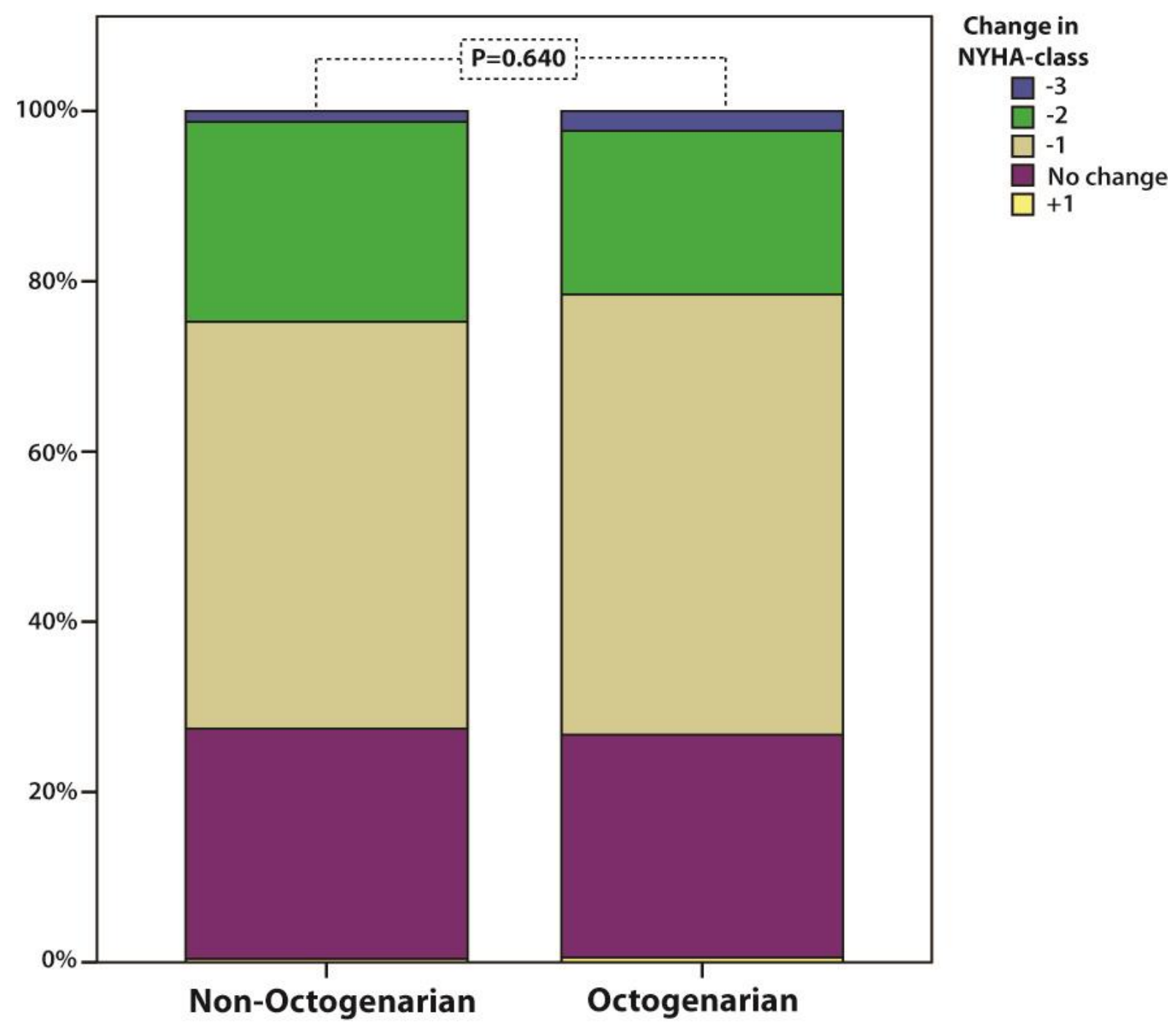


Figure 2: All-cause mortality according to age status compared to an age-matched sample of the general population

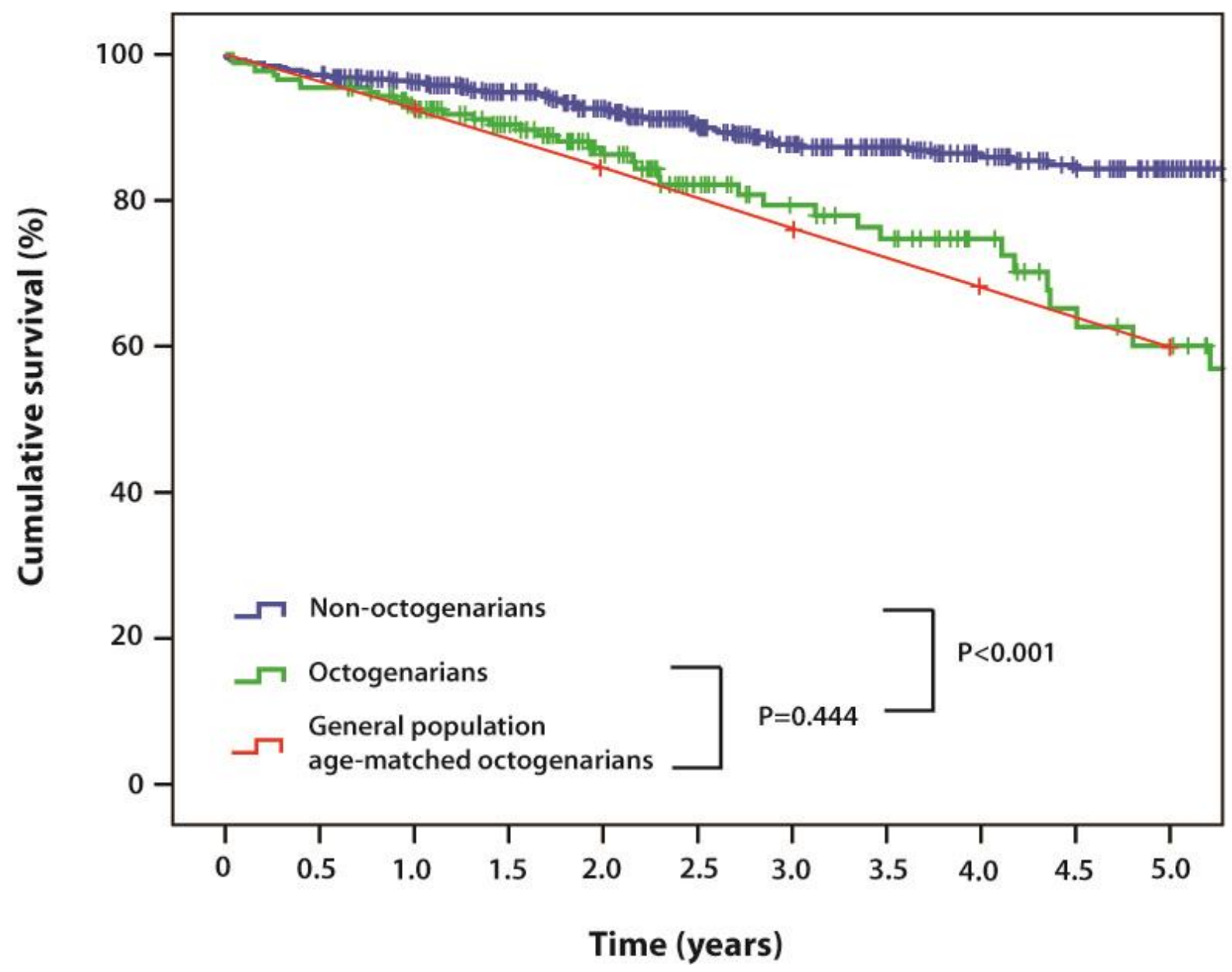


Figure 3: Mode of deaths per group

NON-OCTOGENARIANS $(n=62$ )

Non-cardiac Death ( $\mathrm{N}=31$ ): $50 \%$

Cardiac Death ( $\mathrm{N}=31): 50 \%$

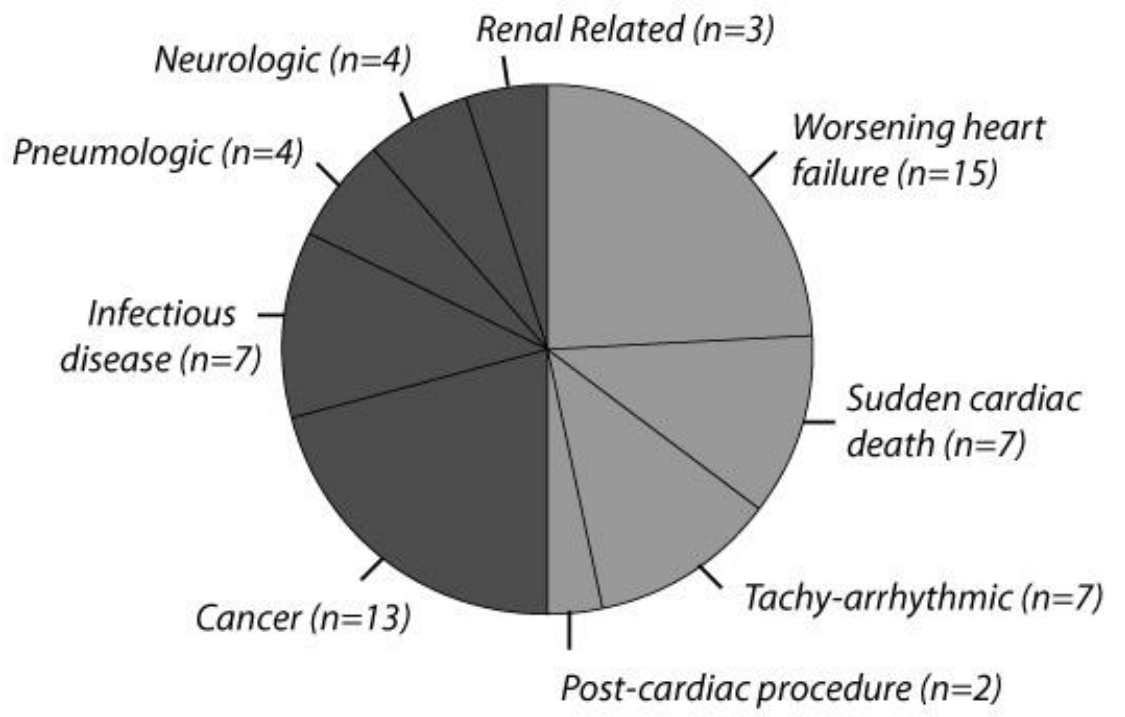

OCTOGENARIANS $(n=38)$

Non-cardiac Death ( $\mathrm{N}=28$ ): 74\%

Cardiac Death ( $\mathrm{N}=10): 26 \%$

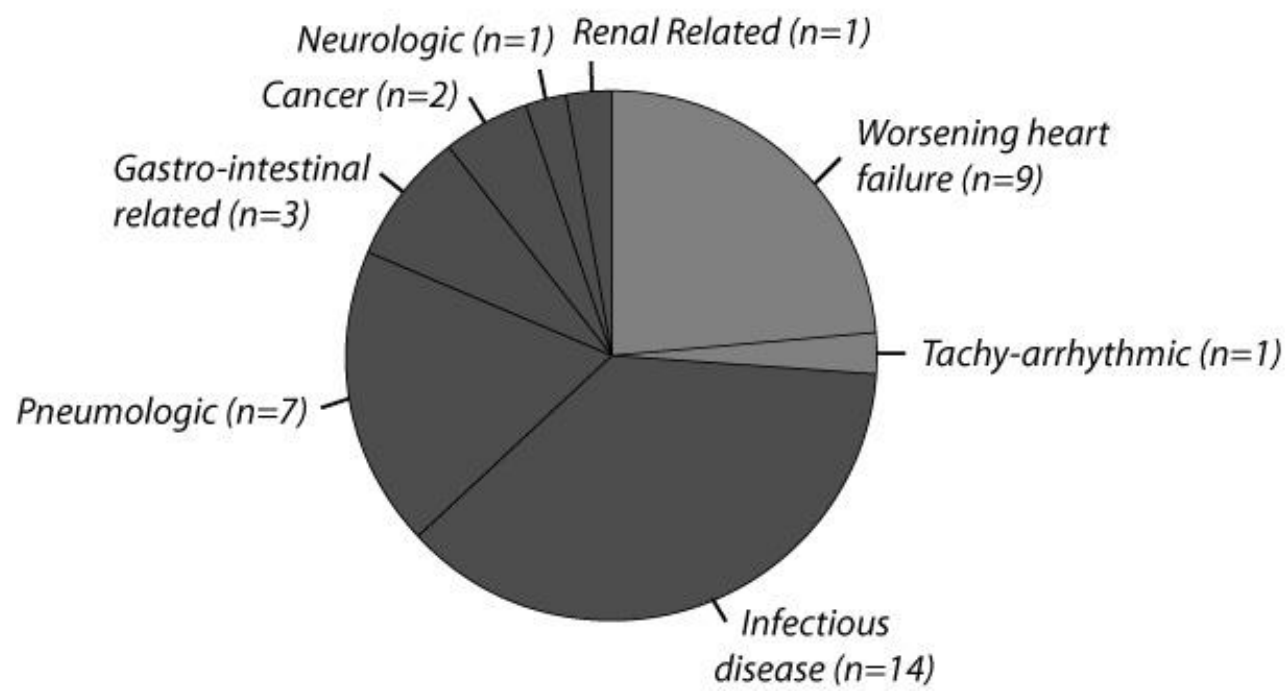


(1) McMurray JJ, Adamopoulos S, Anker SD, Auricchio A, Bohm M, Dickstein K et al. ESC Guidelines for the diagnosis and treatment of acute and chronic heart failure 2012: The Task Force for the Diagnosis and Treatment of Acute and Chronic Heart Failure 2012 of the European Society of Cardiology. Developed in collaboration with the Heart Failure Association (HFA) of the ESC. Eur Heart J 2012;33:1787-1847.

(2) Yancy CW, Jessup M, Bozkurt B, Butler J, Casey DE Jr, Drazner MH et al. 2013 ACCF/AHA guideline for the management of heart failure: a report of the American College of Cardiology Foundation/American Heart Association Task Force on practice guidelines. Circulation 2013;128:e240-e327.

(3) Abraham W, Young JB, Leon AR, Adler S, Bank AJ, Hall SA, Lieberman R et al. Effects of cardiac resynchronization on disease progression in patients with left ventricular systolic dysfunction, an indication for an implantable cardioverter-defibrillator, and mildly symptomatic chronic heart failure. Circulation 2004;110:2864-2868.

(4) Bristow MR,Saxon LA, Boehmer J, Krueger S, Kass D.A, De Marco T et al. Cardiacresynchronization therapy with or without an implantable defibrillator in advanced chronic heart failure. N Engl J Med 2004;350:2140-2150.

(5) Cleland JG, Daubert JC, Erdmann E, Freemantle N, GrasD, Kappenberger L, Tavazzi L, The effect of cardiac resynchronization on morbidity and mortality in heart failure. $N$ Engl J Med 2005;352:1539-1549.

(6) Higgins SL, Hummel JD, Niazi IK, Giudici MC, Worley SJ, Saxon LA et al. Cardiac resynchronization therapy for the treatment of heart failure in patients with intraventricular conduction delay and malignant ventricular tachyarrhythmias. J Am Coll Cardiol 2003;42:1454-1459.

(7) Young JB, Abraham WT, Smith AL, Leon AR, Lieberman R, Wilkoff B et al. Combined cardiac resynchronization and implantable cardioversion defibrillation in advanced chronic heart failure: the MIRACLE ICD Trial. JAMA 2003;289:2685-2694.

(8) Dickstein K, Bogale N, Priori S, Auricchio A, Cleland JG, Gitt A et al. The European cardiac resynchronization therapy survey. Eur Heart J 2009;30:2450-2460.

(9) Go AS, Mozaffarian D, Roger VL, Benjamin EJ, Berry JD, Blaha MJ, Dai SL et al. Heart disease and stroke statistics--2014 update: a report from the American Heart Association. Circulation 2014;129:e28-e292.

(10) Bleeker GB, Schalij MJ, Molhoek SG, Boersma E, Steendijk P et al. Comparison of effectiveness of cardiac resynchronization therapy in patients $<70$ versus $>$ or $=70$ years of age. Am J Cardiol 2005;96:420-422.

(11) Verbrugge F.H, Dupont M, DeVusser P, Rivero-Ayerza M, Van, Herendael H, Vercammen J et al. Response to cardiac resynchronization therapy in elderly patients ( $>/=70$ years) and octogenarians. Eur J Heart Fail 2013;15:203-210.

(12) Kron J, ArandaJ.M.,Jr, MilesW.M, Burkart T.A, Woo G.W, Saxonhouse S.J, Sears S.F, Conti,J.B Benefit of cardiac resynchronization in elderly patients: results from the Multicenter 
InSync Randomized Clinical Evaluation (MIRACLE) and Multicenter InSync ICD Randomized Clinical Evaluation (MIRACLE-ICD) trials. J Interv Card Electrophysiol 2009;25:91-96.

(13) Heidenreich P.A, Tsai, V, Bao,H, Curtis,J, Goldstein,M, Curtis, L et al. Does Age Influence Cardiac Resynchronization Therapy Use and Outcome? JACC Heart Fail 2015;3:497-504.

(14) Suleiman M, Goldenberg I, Haim M, Schliamser JE, Boulos M, Ilan M et al. Clinical characteristics and outcomes of elderly patients treated with an implantable cardioverterdefibrillator or cardiac resynchronization therapy in a real-world setting: data from the Israeli ICD Registry. Heart Rhythm 2014;11:435-441.

(15) Dickstein K, Cohen-Solal A, Filippatos,G, McMurray J.J, Ponikowski P, Poole-Wilson P.A, Stromberg,A. et al. ESC guidelines for the diagnosis and treatment of acute and chronic heart failure 2008: the Task Force for the diagnosis and treatment of acute and chronic heart failure 2008 of the European Society of Cardiology. Developed in collaboration with the Heart Failure Association of the ESC (HFA) and endorsed by the European Society of Intensive Care Medicine (ESICM). Eur J Heart Fail 2008;10:933-989.

(16) Brignole M, Auricchio A, Baron-Esquivias G, Bordachar P, Boriani G, Breithardt OA, Cleland J et al. 2013 ESC Guidelines on cardiac pacing and cardiac resynchronization therapy. Rev Esp Cardiol (Engl Ed) 2014;67:58.

(17) Daubert JC, Saxon L, Adamson PB, Auricchio A, Berger RD, Beshai JF et al. 2012 EHRA/HRS expert consensus statement on cardiac resynchronization therapy in heart failure: implant and follow-up recommendations and management. Europace 2012;14:1236-1286.

(18) Mullens W, Grimm R.A, Verga T, Dresing T, Starling R.C, Wilkoff B.L, Tang WH. Insights from a cardiac resynchronization optimization clinic as part of a heart failure disease management program. J Am Coll Cardiol 2009;53:765-773.

(19) Mullens W, Kepa J., De,Vusser P, Vercammen J, Rivero-Ayerza M, Wagner P et al. Importance of adjunctive heart failure optimization immediately after implantation to improve long-term outcomes with cardiac resynchronization therapy. Am J Cardiol 2011;108:409-415.

(20) Verbrugge FH, Verhaert D, Grieten L, Dupont M, Rivero-Ayerza M, De Vusser P et al. Revisiting diastolic filling time as mechanistic insight for response to cardiac resynchronization therapy. Europace 2013;15:1747-1756.

http://statbel.fgov.be/nl/modules/publications/statistiques/bevolking/downloads/be volking_sterftetafels.jsp 2016. 9-5-2016.

(22) von EE, Altman DG, Egger M, Pocock SJ, Gotzsche PC, Vandenbroucke JP. The Strengthening the Reporting of Observational Studies in Epidemiology (STROBE) statement: guidelines for reporting observational studies. Lancet 2007;370:1453-1457.

(23) Schiller N.B, Shah P.M, Crawford M, DeMaria,A, Devereux R, Feigenbaum H, Gutgesell H. et al. Recommendations for quantitation of the left ventricle by two-dimensional echocardiography. American Society of Echocardiography Committee on Standards, Subcommittee on Quantitation of Two-Dimensional Echocardiograms. J Am Soc Echocardiogr 1989;2:358-367.

(24) Hinkle LE, Jr., Thaler HT. Clinical classification of cardiac deaths. Circulation 1982;65:457-464. 
(25) Martens P, Nijst P, Mullens W. Current Approach to Decongestive Therapy in Acute Heart Failure. Curr Heart Fail Rep 2015;12:367-378.

(26) Linde C, Abraham WT, Gold MR, St John SM, Ghio S, Daubert C. Randomized trial of cardiac resynchronization in mildly symptomatic heart failure patients and in asymptomatic patients with left ventricular dysfunction and previous heart failure symptoms. J Am Coll Cardiol 2008;52:1834-1843.

(27) Ponikowski P, Voors A.A, Anker SD, Bueno H, Cleland JG, Coats AJ et al. 2016 ESC Guidelines for the diagnosis and treatment of acute and chronic heart failure: The Task Force for the diagnosis and treatment of acute and chronic heart failure of the European Society of Cardiology (ESC)Developed with the special contribution of the Heart Failure Association (HFA) of the ESC. Eur Heart J 2016.

(28) Dodson JA, Fried TR, Van Ness PH, Goldstein NE, Lampert R. Patient preferences for deactivation of implantable cardioverter-defibrillators. JAMA Intern Med 2013;173:377-379.

(29) Healey JS, Hallstrom AP, Kuck KH, Nair G, Schron EP, Roberts RS et al. Role of the implantable defibrillator among elderly patients with a history of life-threatening ventricular arrhythmias. Eur Heart J 2007;28:1746-1749.

(30) Mezu U, Adelstein E, Jain S, Saba S. Effectiveness of implantable defibrillators in octogenarians and nonagenarians for primary prevention of sudden cardiac death. Am J Cardiol 2011;108:718-722.

(31) Steinberg BA, Al-Khatib SM, Edwards R, Han J, Bardy GH et al. Outcomes of implantable cardioverter-defibrillator use in patients with comorbidities: results from a combined analysis of 4 randomized clinical trials. JACC Heart Fail 2014;2:623-629.

(32) Marijon E, Leclercq C, Narayanan K, Boveda S, Klug D, Lacaze-Gadonneix J et al. Causes-ofdeath analysis of patients with cardiac resynchronization therapy: an analysis of the CeRtiTuDe cohort study. Eur Heart J 2015;36:2767-2776. 